\title{
Effects of Red Light on the Proliferation Efficiency and Growth of Dendrobium catenatum
}

\author{
Jiangyu Guo \\ The High School Affiliated to Yunnan Normal University, Kunming, China \\ Corresponding Author's Email: 1468163373@qq.com
}

Keywords: Proliferation Efficiency, Red Light, Dendrobium catenatum

\begin{abstract}
Dendrobium catenatum has high medicinal value and is widely used in clinical practice. Due to the special natural growth environment, slow reproduction rate, and the lack of endosperm in its own seeds, the wild population resources are very limited. Artificial breeding uses asexual reproduction of shoot tips, and the culture medium is more mature in the West, and the culture environment is still controversial. The light quality has a unique influence on the growth of $D$. catenatum, so the growth of $D$. catenatum bud tissue culture seedlings was compared under the condition of red light and composite light. We found that the reproductive efficiency of D. catenatum under red light was better than that under composite light, and thus the yield under red light condition also increased. In consequence, red light is more suitable for the tissue culture of $D$. catenatum.
\end{abstract}

\section{Introduction}

Dendrobium catenatum is one of the most precious Chinese herbal medicines. It is ranked as the first of the nine nicest Chinese traditional medical herbs (The other eight herbal medicines are Saussurea involucrata, Panax ginseng, Fallopia multiflora, Poria cocos, Cistanche salsa, Ganoderma Lucidum, Pearl and Cordyceps). The genus Dendrobium belongs to the family Orchidaceae. Orchidaceae is one of the largest and most diverse families of flowering plants, which are distributed all over the world, with more than 28,000 accepted species spanning 763 genera (Christenhusz and Byng, 2016). There are about 74 species of Dendrobium are distributed mainly in the provinces and regions south of the Qinling Mountains in China, especially in southern Yunnan.

Dendrobium is commonly used traditional Chinese medicine and has a long history in medicinal application dating from the late Eastern Han Dynasty in the Shennong Materia Medica. The medicinal functions of $D$. catenatum also have been recorded in many ancient Chinese medical works such as Compendium of Materia Medica and Dictionary of Traditional Chinese Medicine. According to previous researches, the stem of $D$. catenatum contains a variety of alkaloid polysaccharides, amino acids, mucilage and starch, etc. It has the functions of nourishing yin and clearing heat, promoting spleen and stomach, moistening lungs and relieving cough, and improving eyesight. Modern pharmacological studies have shown that Dendrobium is effective in anti-tumor, anti-aging, enhancing immunity, dilating blood vessels and anti-platelet aggregation, and treatging cataracts. Therefore, Dendrobium is widely used in clinical and traditional Chinese medicine 
formulations.

The source of $D$. catenatum for medicinal application has always relied on wild resources. Since the 1970s, the demand for this herb has increased significantly, and wild resources have been unable to meet demand. Due to the extremely harsh requirements of natural ecological conditions, $D$. catenatum has a very low reproductive and growth rate. As early as the 1980s, it was designated as a rare and endangered medicinal plant protected by the state. D. catenatum can have many flowers, while its fruits are relatively few. Each fruit has about 1 million seeds, and the seeds are as fine as dust. The embryos develop without a spherical stage and lack the endosperm tissue, so they must fall into a very suitable environment to germinate. The germination rate under natural conditions is less than 5\%. At first, the seedlings have very limited photosynthetic capacity, so they have to acquire nutrients from the soil with the aid of mycorrhiza fungi. Therefore, the seedling devolops very slowly throughout its life cycle under natural conditions. Also, it has difficulty in sexual reproduction. Nowadays, human can conduct asexual reproduction by using the tissue culture method to overcome all the difficulties mentioned above. The culture medium of MS + auxin is used to satisfy the nutritional condition. The seed or tissue culture of $D$. catenatum can develop into the protocorm and seedling successively in the medium. Therefore, during the tissue culture period, the environmental conditions have a great impact on the tissue culture efficiency and quality. This paper discussed the effect of light quality on seedlings in the tissue culture stage. Red and composite light were used as the variables. Blue and green light had been proved to be inefficient, so they were not included. Our results showed that red light was more beneficial to the development of shoot tip, and the seedling quality under red light was also better than that under composite light, so red light should be applied more in production of $D$. catenatum.

\section{Materials and Methods}

\subsection{Experimental Materials and Processing}

The experiment was conducted from February 15 to October 15 in 2017. The tissue culture was carried out in a thermostatic cabinet illuminated with a lamp tube, and the temperature was basically controlled at $25^{\circ} \mathrm{C} \pm 3^{\circ} \mathrm{C}$. The humidity in the box is kept at $50 \%$, the light is made of energy-saving lamps, the light intensity is $1600-2000$ lux, the white light temperature is $6000 \mathrm{~K}$, the red light temperature is $4500 \mathrm{~K}$, and the daily light time is adjusted according to the growth condition. The initial average is $4.5 \mathrm{~h} / \mathrm{d}$, which gradually increases to $8 \mathrm{~h}$. $/ \mathrm{d}$.

The experimental medium was MS $+2 \mathrm{mg} / \mathrm{L}$ peptone $+0.4 \mathrm{mg} / \mathrm{LMAA}$, using $20 \mathrm{~g}$ of sucrose as a carbon source. The $\mathrm{pH}$ was adjusted to 5.8 and equipped with activated carbon powder. All the medium was used after autoclaving.

The $D$. catenatum buds were disinfected with mercury and the inoculation tools were disinfected with alcohol for $5 \mathrm{~min}$ before inoculation. The whole process of inoculation was carried out using an alcohol lamp in a super-clean workbench. Once completed, the culture bottles were labeled and placed in a culture chamber corresponding to the light quality.

In order to prevent pollution, measurements were made only twice throughout the whole process. After that, the medium is renewed.

\subsection{Comparative Experiment on the Efficiency of Tissue Culture under Different Light Qualities}

The shoot tips were obtained from the tissue cultured seedlings of $D$. catenatum, and then were inoculated into 16 bottles with medium and divided into two groups of 8 bottles for each. Each bottle was inoculated with 10M2 bud tip tissue, which was cultivated under different light. The 
initial observation was performed every two weeks, and the later observation was conducted after one month. The number of seedlings, the number of leaves, the number of leaves and the number of protocorms were determined, and the height of stems was finally measured. The effects of different light on the tissue culture efficiency of $D$. catenatum were compared.

\section{Results and Discussion}

\subsection{Discussion of Research}

Our data showed that the proliferation efficiency of $D$. catenatum under red light is higher. The main reason would be confered that the shoot tip is the site where most plants produce plant hormones, especially auxin. Normally, the blue-violet band is easy to inhibit auxin production by accelerating the auxin decomposition or stimulating the growth of the auxin inhibitor in the plant body. Red light does not contain the blue-violet high-energy light band, so it can be inferred that the red light is more effective in the induction of auxin secretion. Auxin is one of the essential components for promoting shoot growth and root growth. Although different growth line concentrations have different gain effects on the growth of different parts, this endogenous phytohormone is likely to act synergistically or even promote exogenous auxin analogs (NAA in this medium) to promote growth. However, in the experiment, the growth of the red light treatment seedlings in the early stage was slow, and the red light on the further development of the original bulbs was inhibited. Some of the original bulbs were yellow and stagnation occurred. It may be caused by an excessive concentration of auxin. At the same time, it was observed that although the growth of tissue culture seedlings under composite light treatment was rapid, the difference was large, and the stems were generally thinner, with more dead leaves and slower growth rate. This may also be the result of auxin inhibition by the blue-violet band in the composite light, resulting in insufficient stem development.

\subsection{Experimental Result}

Our data (Table 1, Fig 1 to 5) showed that red light has higher tissue culture efficiency of $D$. catenatum, and is more beneficial to the production of $D$. catenatum with better quality. The stem height is better, the dead leaf and aging seedlings are less. However, the number of shoots under composite light is more, and the original bulbs are also better developed. In summary, it is suitable to use composite light for the pretreatment of $D$. catenatum, and then transferred the seedlings to red light conditions for further cultivation.

Tab 1 Light quality Time according to colorless and red.

\begin{tabular}{|c|c|c|c|c|}
\hline \multirow{2}{*}{$\begin{array}{c}\text { Light quality } \\
\text { Time }\end{array}$} & \multicolumn{2}{|c|}{ Colorless } & \multicolumn{2}{c|}{ Red } \\
\cline { 2 - 5 } & Plant height & $\begin{array}{c}\text { Stem } \\
\text { thickness }\end{array}$ & Plant height & $\begin{array}{c}\text { Stem } \\
\text { thickness }\end{array}$ \\
\hline May 2 & 3.8 & 0.238 & 3.988 & 0.241 \\
\hline October 17 & 5.209 & 0.202 & 5.255 & 0.309 \\
\hline
\end{tabular}




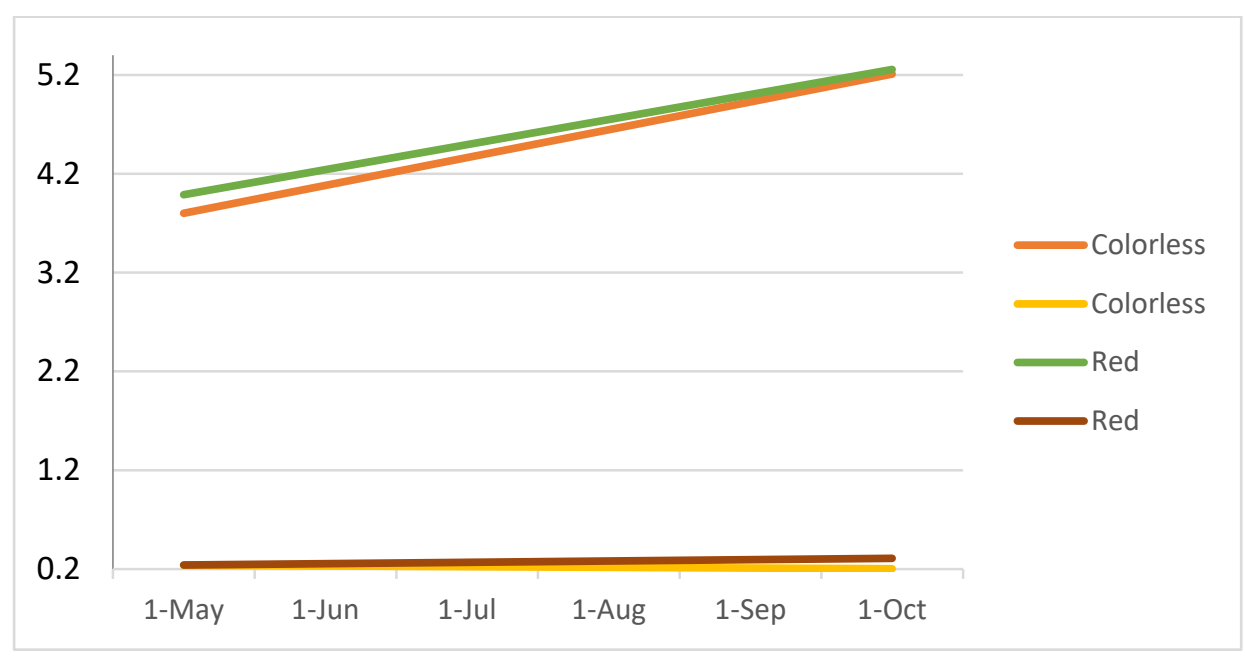

Fig 1 stem height/thickness broken line diagram of Dendrobium germinum tissue culture in different light

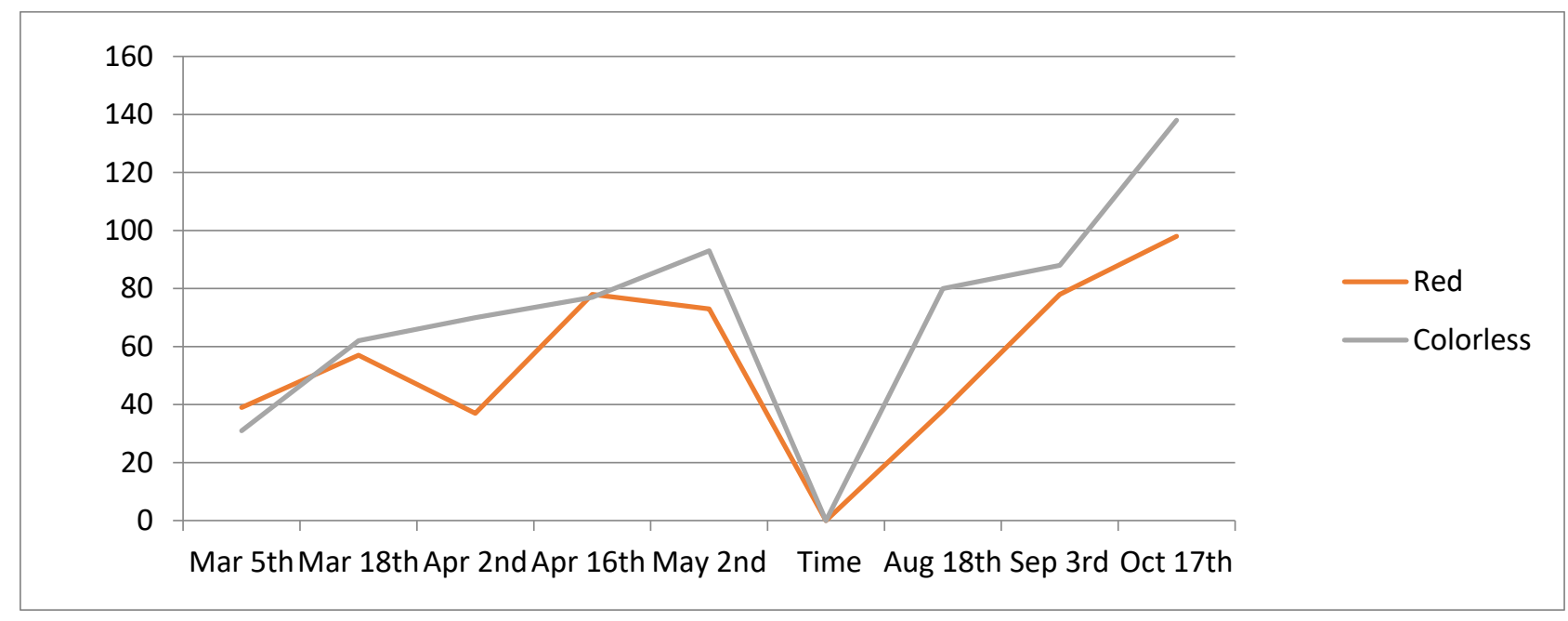

Fig 2 Statistics of seedling number of Dendrobium germination tissue culture under different light quality

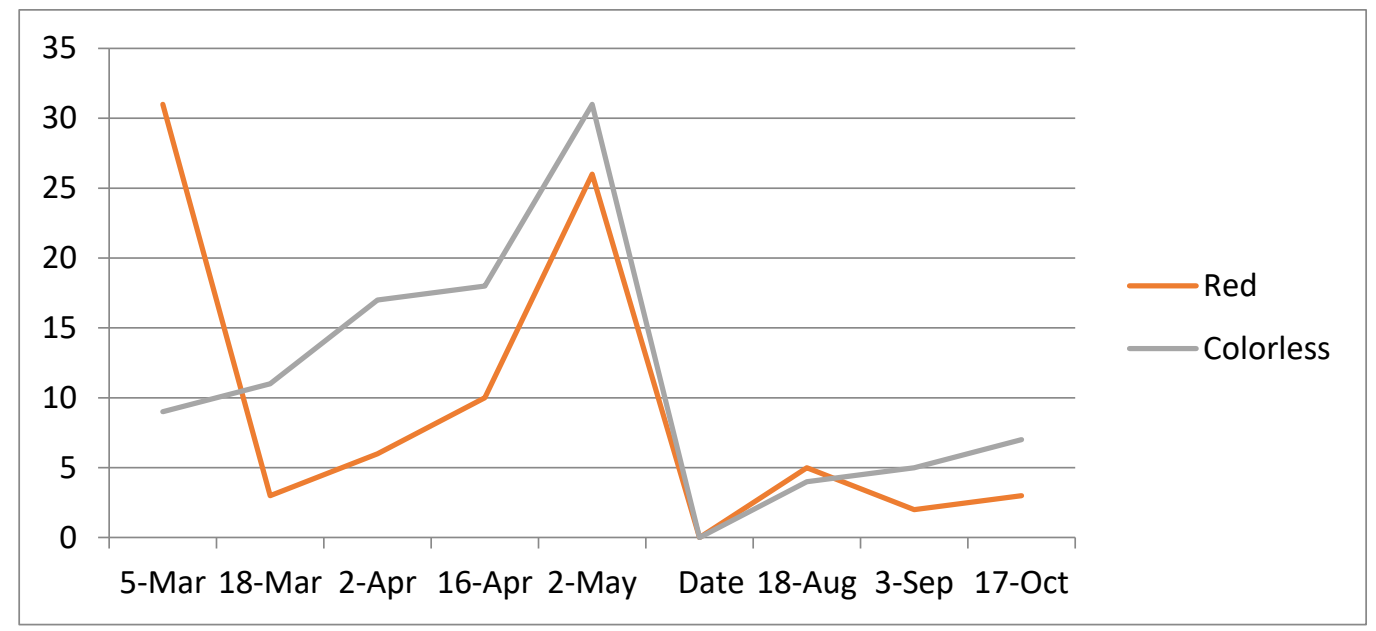

Fig 3 Statistical broken line diagram of protocorm number of budding tissue culture of Dendrobium under different photoplasm 


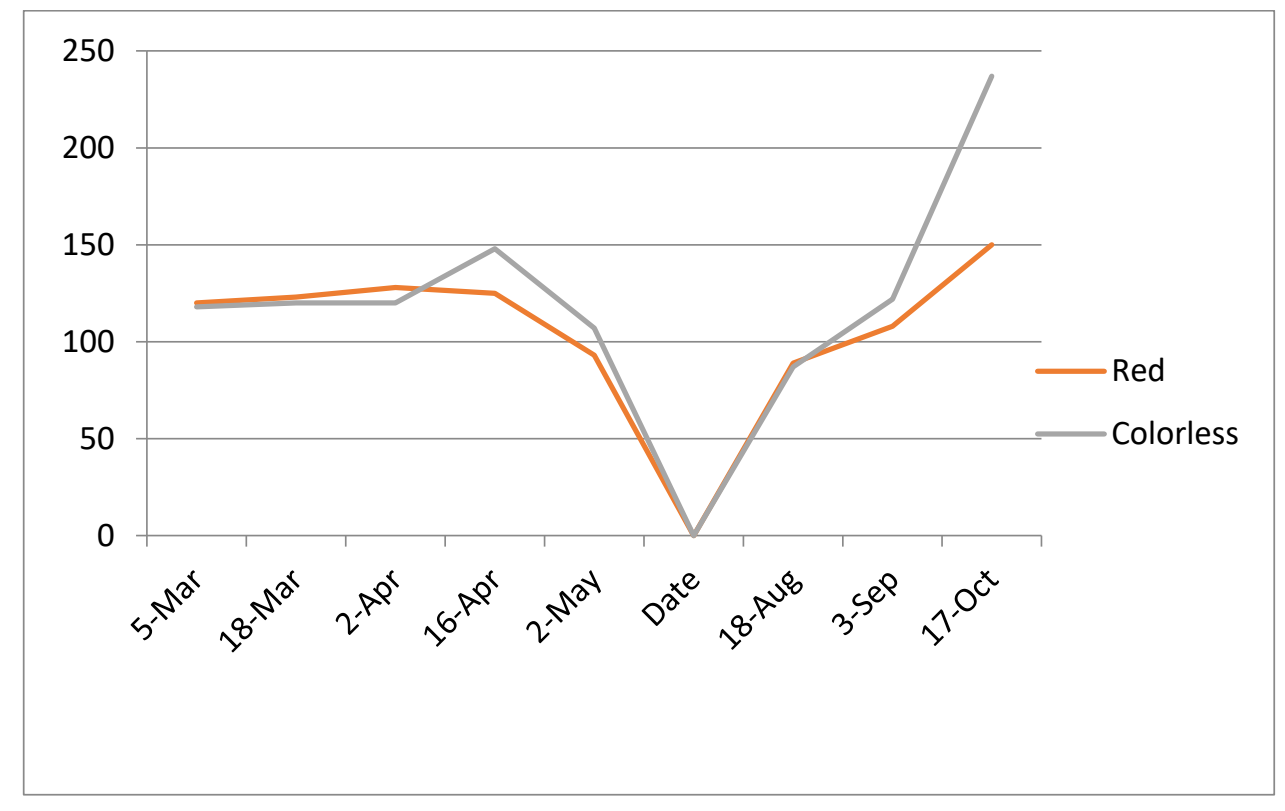

Fig 4 Leaf number statistics of Dendrobium budding tissue culture under different light

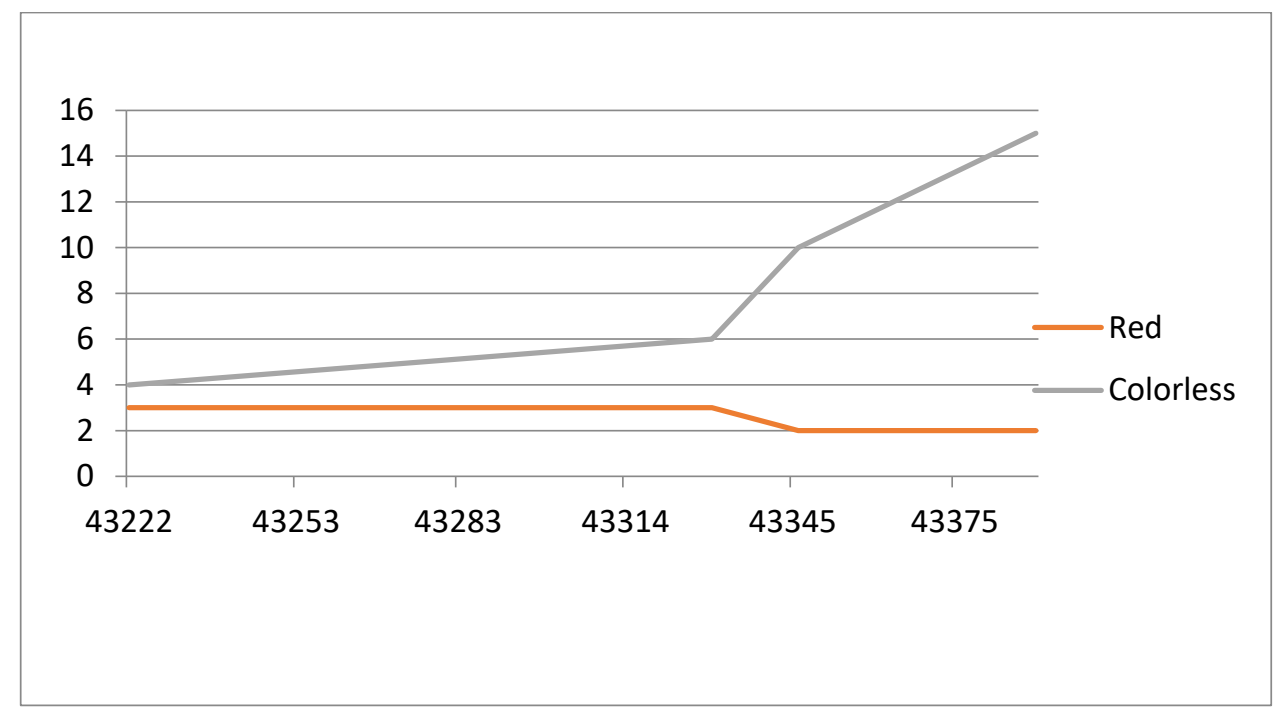

Fig 5 Statistics of leaf seedling number in tissue culture of Dendrobium with different light quality

\section{Acknowledgments}

I wish to thank Professor Hong Hu (Kunming Institute of Botany, Chinese Academy of Science) for technical support.

\section{References}

[1] Christenhusz, M.J.M., Byng, J.W., 2016. The number of known plant species in the world and its annual increase. Phytotaxa 261, 201-217.

[2] Enhanced-micropropagation-of-Dendrobium-huoshanense-C-Z-Tang-et-S-J-Cheng-through-protocorm-like-bodiesThe-effects-of-cytokinins-carbohydrate-sources

[3] Effects of different hormone ratio on rooting of Dendrobium catenatum tissue seedling _ Zhu Lina

[4] Effects of different hormone ratios on tissue culture of Dendrobium catenatum _ Chen Zhaogui

[5] Optimization of tissue culture and rapid propagation of dendrobium catenatum 